\title{
Les Eyzies-de-Tayac-Sireuil - Abri de Crabillat « Moulin de Crabillat »
}

$n^{\circ} 026236$

André Morala et Catherine Cretin

\section{(2) OpenEdition \\ Journals}

Édition électronique

URL : http://journals.openedition.org/adlfi/16386

ISSN : 2114-0502

Éditeur

Ministère de la culture

Référence électronique

André Morala et Catherine Cretin, « Les Eyzies-de-Tayac-Sireuil - Abri de Crabillat « Moulin de

Crabillat » », ADLFI. Archéologie de la France - Informations [En ligne], Aquitaine, mis en ligne le 10 février 2016, consulté le 03 mai 2019. URL : http://journals.openedition.org/adlfi/16386

Ce document a été généré automatiquement le 3 mai 2019

(c) Ministère de la Culture et de la Communication, CNRS 


\title{
Les Eyzies-de-Tayac-Sireuil - Abri de Crabillat « Moulin de Crabillat »
}

$n^{\circ} 026236$

\author{
André Morala et Catherine Cretin
}

Lien Atlas (MCC) :

http://atlas.patrimoines.culture.fr/atlas/trunk/index.php?

ap_theme=DOM_2.01.02\&ap_bbox=0.976;44.900;1.117;44.967

Dans la perspective de nouvelles datations radiométriques permettant de préciser la chronologie du faciès du Magdalénien à triangles scalènes, un programme de sondage mené dans le but de prélever des échantillons osseux a été réalisé sur le site du Moulin de Crabillat.

2 Les deux tentatives de datation précédentes effectuées sur le matériel provenant des fouilles de D. et E. Peyrony (Peyrony D., Peyrony E., 1941) conservé au musée national de la Préhistoire, n'ayant pas donné de résultat satisfaisant, nous avons donc décidé d'intervenir directement sur le site pour effectuer de nouveaux prélèvements.

3 Cependant cette opération, qui dépendait nécessairement de la conservation de dépôts encore en place suffisamment bien préservés, n'était pas garantie, car les fouilles Peyrony remontaient à la fin des années trente et le site, jamais ré-exploité depuis, demeurait dans un total état d'abandon.

4 Le premier travail a donc été de tenter de situer précisément sur le terrain la zone fouillée, d'en déterminer l'emprise, puis de rechercher d'éventuels vestiges archéologiques épargnés du niveau ayant livré l'industrie magdalénienne.

5 À notre satisfaction, un témoin stratigraphique de quelques mètres carrés et une petite coupe frontale de l'époque des premières fouilles subsistaient encore dans le prolongement d'un petit abri situé à l'est de la parcelle. Bien que passablement dégradés par l'érosion et par quelques sapes clandestines, ces dépôts ont néanmoins révélé l'existence de témoins anthropiques correspondant au niveau recherché. 
6 La zone archéologique se situe à la verticale de la grande falaise qui, orientée au sud, surplombe la vallée de la Beune à environ $20 \mathrm{~m}$ à l'est des ruines de constructions annexes au moulin. Cette zone, où furent réalisées les recherches, coïncide assez bien avec la description qu'en donnent les Peyrony dans leur publication (Peyrony D., Peyrony E., op. cit.). Ainsi nous avons pu circonscrire les quelques $20 \mathrm{~m}^{2}$ de surface dégagée lors de ces fouilles qui forment un replat en légère pente vers le sud.

7 À l'arrière, contre la paroi, s'appuie la petite coupe frontale signalée précédemment, prolongée à sa gauche par le petit abri formant actuellement un creux sous la falaise d'environ $2 \mathrm{~m}$.

8 À droite de la coupe, l'espace est occupé par le témoin sédimentaire qui, en fait, correspond à une réserve stratigraphique d'un peu plus de $6 \mathrm{~m}^{2}$ de surface sur environ 1,30 m d'épaisseur de dépôts, volontairement conservée en place par les Peyrony. En effet, cette indication nous a été apportée par la découverte de perforations dans le rocher et de scellements de piquets métalliques retrouvés tout autour, indiquant qu'il y avait eu la volonté de la part des chercheurs de protéger la stratigraphie du gisement et d'en assurer la conservation (cf. fig). Ce témoin se situe perpendiculairement à la falaise formant une saillie de 3,50 $\mathrm{m}$ en avant de la paroi rocheuse. Il présente deux belles coupes que nous avons pu remettre en état et étudier ; l'une orientée à l'ouest, l'autre au sud.

9 La stratigraphie synthétique générale est la suivante : le substratum calcaire coniacien est directement recouvert de plusieurs dizaines de centimètres de dépôts argileux localement indurés par les carbonates provenant du karst, contenant de petits blocs calcaires et des cailloux dispersés. La partie inférieure de ces dépôts renferme l'unité archéologique de base (NA base). Cet ensemble est ensuite surmonté par un cailloutis à matrice plus ou moins sableuse, siliceuse, ou limoneuse d'une cinquantaine de centimètres, au sein duquel quelques éléments anthropiques ont été observés (NA sommet). Cette formation composée de blocaille, s'enrichit progressivement vers le haut avec une tendance à l'augmentation progressive du volume des éléments. Ainsi, le sommet du témoin Peyrony est-il coiffé par un énorme bloc détaché de la falaise, dont l'emplacement d'origine est encore visible contre la paroi. Des marques d'aménagements troglodytiques de l'abri, en relation ou pas avec le moulin, sont visibles, sur le gros bloc effondré (trou de poteau) comme sur la paroi (larmiers, trous de boulins...).

10 Concernant les indices archéologiques, ils ont été localisés dans les divers endroits de la zone d'intervention. Sur la terrasse aménagée par les fouilles précédentes, le nettoyage des dépôts superficiels remaniés a fourni du matériel archéologique minéral et osseux. Il conviendra à présent de vérifier si ce matériel, ainsi que celui publié par D. et E. Peyrony appartiennent à un seul et même niveau archéologique ou s'il s'agit d'une association industrielle de plusieurs niveaux magdaléniens de stades différents.

11 Malgré tout l'intérêt des découvertes et des observations faites, le point le plus positif de l'opération reste toutefois la découverte de témoins stratifiés en place dans le remplissage permettant le prélèvement d'échantillons osseux à dater.

12 Deux unités archéologiques ont donc été identifiées, distinctes l'une de l'autre tant au niveau altimétrique que stratigraphique.

13 À l'exception d'une petite quantité d'objets (lithiques et osseux assez mal conservés) mise en évidence dans la partie médiane de la coupe centrale, attribuée à l'ensemble archéologique supérieur (NA sommet), la majorité du matériel anthropique découvert appartient à l'unité archéologique inférieure (NA base) qui repose directement sur le 
substratum calcaire coniacien. D'ouest en est, ce dernier niveau a été observé dans les dépôts argileux de la partie la plus abritée par le rocher et de la base de la coupe centrale, ainsi qu'au bas de la coupe ouest et de la coupe sud. Dans ce secteur, et plus particulièrement à la base de la coupe ouest, les témoins d'occupation de cette unité archéologique (silex, os, plaquettes calcaires rubéfiées...), bien représentés, sont contenus dans les sédiments bruns très foncés à noirs charbonneux d'une aire de combustion (correspondant sans doute au foyer dont faisaient état les Peyrony dans leur publication).

Bien que constitués de fragments d'os de dimensions modestes (quelques centimètres), une quinzaine d'échantillons, d'un assez bon état de conservation en apparence, ont été extraits dans ce niveau. Après examen, certains d'entre eux ont été sélectionnés pour la réalisation des datations.

Le matériel lithique, bien que limité en nombre, est très satisfaisant, car il fournit, en parfaite association stratigraphique et avec une très bonne cohérence taphonomique, plusieurs triangles scalènes (marqueurs de ce faciès industriel) venant conforter de manière incontestable la position des éléments osseux à dater, ce qui devrait assurer la bonne fiabilité des résultats attendus.

L'objectif principal de l'opération a donc été atteint, les analyses radiocarbone seront à présent déterminantes. Nous terminons cet état des travaux menés sur le gisement du Moulin de Crabillat en faisant mention d'une dernière réalisation que nous avons estimée nécessaire : avec l'accord des propriétaires du site, les coupes stratigraphiques ouest et sud du témoin Peyrony ont été murées pour assurer la préservation des derniers vestiges stratigraphiques subsistants. Cet ultime témoin stratigraphique du gisement de Crabillat bénéficie à présent à nouveau d'une protection physique.

17 Avec la collaboration de Ferullo Olivier, Le Fillâtre Virginie, Madelaine Stéphane, Maumont Michel
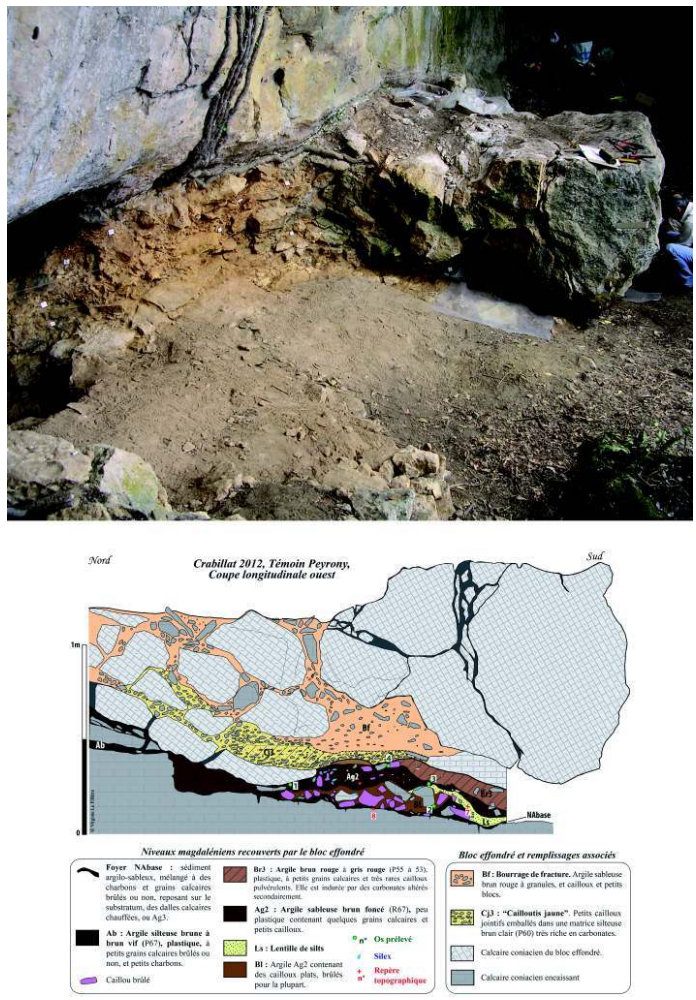
Fig. 1 et 2

En haut : vue générale du gisement (de gauche à droite) abri, coupe frontale et «Témoin Peyrony». Cliché : A. Morala, MNPE.

\section{BIBLIOGRAPHIE}

Peyrony, D. et Peyrony E., « Gisement préhistorique de Crabillat. Ses rapports avec les dépôts à formes géométriques du Paléolithique supérieur et du Mésolithique », Bulletin de la Société Préhistorique Française, t. 38, nº 12, 1941, p. 245-262.

INDEX

Index géographique : Aquitaine, Dordogne (24), Les-Eyzies-de-Tayac-Sireuil Index chronologique : Magdalénien operation Sondage (SD)

Mots-clés : triangles scalènes, ossement

\section{AUTEURS}

\section{ANDRÉ MORALA}

MCC 